\title{
A LIMIT RESULT ASSOCIATED WITH CERTAIN RANDOM TWO-POINT BOUNDARY VALUE PROBLEMS
}

\author{
G. $\mathrm{YIN}^{1}$, G. ZHAO ${ }^{2}$ \\ ${ }^{1,2}$ Department of Mathematics \\ Wayne State University \\ Detroit, MI 48202 GE Global Research Niskayuna, NY 12309, USA
}

\begin{abstract}
A nonlinear differential equation with wide-band random noise and random boundary conditions is considered in this work. Our main effort is on obtaining a diffusion limit using weak convergence methods. The limit can be used as approximation to the original problem leading to reduction of computational complexity.
\end{abstract}

AMS Subject Classification: 60F05, 60J60, 34B99

Key Words: two-point boundary value problem, random perturbation, wide-band noise, diffusion process, weak convergence

Received: $\quad$ May 22, 2017; Accepted: October 24, 2017;

Published: January 4, 2018 doi: 10.12732/dsa.v27i1.2

Dynamic Publishers, Inc., Acad. Publishers, Ltd. https://acadsol.eu/dsa

\section{INTRODUCTION}

Applications in a wide variety of fields require the handling of two-point boundary value problems. Many problems involving partial differential equations with boundary conditions are reduced to such problems, for example, the well-known classical Sturm-Liouville problems. Such problems also arise in many control and optimization systems. The widely used LQG (linear quadratic Gaussian regulator) control problems often lead to the problem of solving a Riccati equation with boundary conditions. Moreover, because of the need in modeling of complex and real-world systems, one is forced to take random noise into consideration.

In this paper, we consider the following random two-point boundary value prob- 
lem:

$$
\begin{gathered}
\ddot{y}^{\varepsilon}(t)=\frac{1}{\varepsilon} F\left(y^{\varepsilon}(t), \dot{y}^{\varepsilon}(t), \xi^{\varepsilon}(t)\right) \\
y^{\varepsilon}(0)=\beta_{1}^{\varepsilon}, \quad y^{\varepsilon}(1)=\beta_{2}^{\varepsilon} .
\end{gathered}
$$

This paper is concerned with the limit associated with this type of boundary value problems. For notation simplicity, we shall assume that $y^{\varepsilon}(\cdot), \xi^{\varepsilon}(\cdot)$ are real-valued stochastic processes, and $\beta_{i}^{\varepsilon}, i \leq 2$ are real-valued random variables throughout the paper. However, all the subsequent development may be extended to multidimensional cases. In (1), $\varepsilon>0$ is a small parameter. We are particularly interested in investigating the asymptotic behavior of the underlying system, and getting a limit theorem as $\varepsilon \rightarrow 0$.

Several people tackled random boundary value problems from different angles; see for example, [18], [17], and [16], among others; see also related paper for deterministic problems [4] for instance. Two point boundary value problems of nonlinear equations with deterministic boundary conditions was studied in White and Franklin [18]. Linear and nonlinear deterministic ordinary differential equations with random boundary conditions were treated in Xia, Boyce, and Barry [17]. Random boundary value problems in conjunction with the determination of joint probability density distribution for the solution was developed in Xia [16].

Our main reference in this paper is [18], in which White and Franklin treated the following boundary value problem:

$$
\begin{gathered}
\ddot{y}^{\varepsilon}(t)=F\left(y^{\varepsilon}(t), \dot{y}^{\varepsilon}(t), \xi^{\varepsilon}(t)\right) \\
y^{\varepsilon}(0)=0, \quad y^{\varepsilon}(1)=1 .
\end{gathered}
$$

In lieu of dealing with (3)-(4) directly, an alternative approach-the method of shooting parameter was employed.

Choose a shooting parameter $\alpha^{\varepsilon}$, and replace the boundary condition (4) by the initial condition

$$
y^{\varepsilon}(0)=0, \dot{y}^{\varepsilon}(0)=\alpha^{\varepsilon} .
$$

Let the solution of (3) and (5) be denoted by $y^{\varepsilon}\left(t ; \alpha^{\varepsilon}\right)$. The solution of (3)-(4) is then obtained for random variables $\alpha^{\varepsilon}$ satisfying the following nonlinear boundary condition

$$
y^{\varepsilon}\left(1 ; \alpha^{\varepsilon}\right)=1
$$

It was shown that with large probability, there is a solution to this problem. Moreover, let $y^{0}(t, \alpha)$ be the solution of the "averaged" system, then the normalized deviation

$$
\frac{1}{\sqrt{\varepsilon}}\left(\begin{array}{c}
y^{\varepsilon}(t)-y^{0}(t) \\
\dot{y}^{\varepsilon}(t)-\dot{y}^{0}(t)
\end{array}\right)
$$


converges weakly to a diffusion process.

In [16], the following problem was investigated:

$$
\begin{aligned}
& \dot{y}^{\varepsilon}(t)=F\left(y^{\varepsilon}(t), \xi^{\varepsilon}(t)\right), \\
& A y^{\varepsilon}(0)+B y^{\varepsilon}(1)=\alpha^{\varepsilon},
\end{aligned}
$$

where $\xi^{\varepsilon}(t)=\xi_{0}(t)+\varepsilon \xi_{1}(t)+\varepsilon^{2} \xi_{2}(t)+\cdots$. By means of solving a sequence of deterministic partial differential equations, the density function was determined.

In this work, the framework is different from the aforementioned papers. Although our formulation is somewhat similar to that of [18], there are main differences. To put into perspective and to see what is new in the current paper, we begin by noting that the problem considered in this paper is very different from that of [18]. Seemingly having similar form, the right-hand side of (1) is $\varepsilon^{-1} F\left(y^{\varepsilon}(t), \dot{y}^{\varepsilon}(t), \xi^{\varepsilon}(t)\right)$, whereas the right-hand side of $(3)$ is $F\left(y^{\varepsilon}(t), \dot{y}^{\varepsilon}(t), \xi^{\varepsilon}(t)\right)$. Thus (3) can be considered as a "small" perturbation about a deterministic ODE, whereas (1) is a "large" perturbation leading to a limit SDE. The subtle difference due to the addition of the term $\varepsilon^{-1}$ is that as $\varepsilon \rightarrow 0$, a limit ordinary differential equation is obtained in [18], whereas in this paper, as $\varepsilon \rightarrow 0$, the limit is a diffusion. To the best of our knowledge, such a more "singularly" perturbed boundary value problem has not been considered in the literature previously. As $\varepsilon$ is getting smaller and smaller, there are more and more fluctuations. However, contrary to one's intuition, the system does not blow up as $\varepsilon \rightarrow 0$. It is interesting to note that because of the $\varepsilon^{-1}$ used, the limit has a "correction" term similar in spirit to the Wong-Zakai approximation, whereas the error term considered in [18] follows the work of Khasminskii [6]. Moreover, the techniques used in this paper is based on the more up-to-dated martingale averaging methods. The martingale averaging methods have been developed by Kushner; see for example, [11], which have been developed further and used in stochastic approximation for a wide range of systems in Kushner and Yin [12]. In this paper, our effort is devoted to reduction of complexity. The original problem with wideband noise and small parameter $\varepsilon$ is difficult to deal with. The limit diffusion system is much simpler. Thus, we may use the limit system as a good approximation to the original system. We shall study the two-point boundary value problem from a weak convergence point of view. By using recent results in diffusion approximation, the randomly perturbed two-point boundary value problem is approximated by that of a limit diffusion process. In lieu of using a "white noise" model, we consider a wide-band noise model, which is more realistic in various applications. Roughly speaking, a wide-band noise is one such that it approximates the "white noise". To be more specific, let $R^{\varepsilon}(s)$ be the correlation of $\xi^{\varepsilon}(\cdot)$, i.e.,

$$
R^{\varepsilon}(s)=E \xi^{\varepsilon}(t+s) \xi^{\varepsilon}(t)
$$


and let $S^{\varepsilon}(\cdot)$ be the power spectral density (we assume it exists) $S^{\varepsilon}(\mu)=\int_{-\infty}^{\infty} e^{i \mu s}$ $R^{\varepsilon}(s) d s$. If $\xi(\cdot)$ is wide-band noise, then $S^{\varepsilon}(\mu)$ is band limited, i.e., $S^{\varepsilon}(\mu)=0$ for $|\mu|>\mu_{0}$, for some $\mu_{0}>0$, and the interval $\left[-\mu_{0}, \mu_{0}\right]$ is wide enough. In fact, we shall assume $\xi^{\varepsilon}(t)=\xi\left(t / \varepsilon^{2}\right)$. Consequently, the correlation function is $R^{\varepsilon}(s)=R(s / \varepsilon)$ and its spectral density is $S^{\varepsilon}(\mu)=\varepsilon S(\varepsilon \mu)$. The bandwidth is of the order $1 / \varepsilon$. As $\varepsilon$ is getting smaller and smaller, the bandwidth is getting wider and wider; and the spectral of $\frac{1}{\varepsilon} \xi\left(\frac{t}{\varepsilon^{2}}\right)$ tends to that of white or Gaussian noise as $\varepsilon \rightarrow 0$.

As mentioned before, our main goal is to obtain a limit theorem by means of weak convergence methods. For definitions as well as general theory of weak convergence, the readers are referred to [1], [11], and [3]. Terms as weak convergence, tightness, Skorohod topology etc. will be used without specific mention.

To proceed, we assume that $\xi^{\varepsilon}(\cdot)$ is exogenous in the sense, for each $t$ and each set $G$ in $\sigma\left(\xi^{\varepsilon}(u) ; u>t\right), P\left(G \mid \xi^{\varepsilon}(u) ; u \leq t\right)=P\left(G \mid \xi^{\varepsilon}(u), x^{\varepsilon}(u) ; u \leq t\right)$. The symbol $K$ denotes a generic positive constant, its value may change from usage to usage however; $C_{0}^{2}$ denotes the set of $C^{2}$ functions with compact support, and $D^{r}[0, \infty)$ denotes the space of functions which live in $\mathbb{R}^{r}$, and which are right continuous with left-hand limits endowed with the Skorohod topology; $E_{t}^{\varepsilon}$ denotes the conditioning on the $\sigma$-algebra $\sigma\left(\xi^{\varepsilon}(u) ; u \leq t\right)$; " $\Rightarrow$ " means "weak convergence."

The organization of the remainder of the paper is as follows. The methods of shooting parameters is discussed next, and the main theorem is stated. The proof of the theorem is then presented in Section 3. Some examples are given in Section 4. Finally, some concluding remarks are made in Section 5.

\section{LIMIT RESULT}

Solving boundary value problems by means of shooting parameter methods were discussed extensively in [5], for instance. In light of [18], [16], and [5], instead of treating the boundary value problem (1)-(2), we consider the following initial value problem.

$$
\begin{gathered}
\ddot{y}^{\varepsilon}(t)=\frac{1}{\varepsilon} F\left(y^{\varepsilon}(t), \dot{y}^{\varepsilon}(t), \xi^{\varepsilon}(t)\right) \\
y^{\varepsilon}(0)=\beta_{1}^{\varepsilon}, \quad \dot{y}^{\varepsilon}(0)=\gamma^{\varepsilon} .
\end{gathered}
$$

where $\gamma^{\varepsilon}$ is a random variable.

We begin by assuming that $F(\cdot, \cdot)$ is smooth enough, and that for each $\varepsilon>0$, there is a unique solution (unique in the sense of in distribution) for (7)-(8). Because of our main focus here is the limit property, it is more instructive to concentrate on and emphasize on the weak convergence aspect. Note that regarding the solution to (7)-(8), the uniqueness here is only assumed in the sense of in distribution because that we only need to work with probability measures. The rational is that the problem 
with a small parameter is more difficult to deal with in general. Rather than treating this problem, we can examine the limit process so as to substantially simplify the calculations. Also as mentioned before, the limit in our case is no longer an ordinary differential equation but rather a stochastic differential equation. We would also like to add that in recent years, more analysis methods and techniques have been developed to analyze singularly perturbed diffusions; see for example, the work of Khasminskii and Yin $[7,8,9]$ and references therein. In addition, for recent results on the existence and uniqueness of solutions of boundary value problems, we refer to [14] and references therein.

Denote the solution by $y^{\varepsilon}\left(t ; \gamma^{\varepsilon}\right)$. The solution of the original boundary value problem is obtained by choosing $\gamma^{\varepsilon}$ such that

$$
y^{\varepsilon}\left(1 ; \gamma^{\varepsilon}\right)=\beta_{2}^{\varepsilon}
$$

We define $x_{1}^{\varepsilon}(t)=y^{\varepsilon}(t), x_{2}^{\varepsilon}(t)=\dot{y}^{\varepsilon}(t)$. Then (7)-(8) can be written as:

$$
\begin{gathered}
\left(\begin{array}{l}
\dot{x}_{1}^{\varepsilon}(t) \\
\dot{x}_{2}^{\varepsilon}(t)
\end{array}\right)=\left(\begin{array}{l}
x_{2}^{\varepsilon}(t) \\
\frac{1}{\varepsilon} F\left(x^{\varepsilon}(t), \xi^{\varepsilon}(t)\right)
\end{array}\right) \\
\left(\begin{array}{c}
x_{1}^{\varepsilon}(0) \\
x_{2}^{\varepsilon}(0)
\end{array}\right)=\left(\begin{array}{c}
\beta_{1}^{\varepsilon} \\
\gamma^{\varepsilon}
\end{array}\right)
\end{gathered}
$$

To proceed, the following assumptions are needed.

(A1) $\xi(\cdot)$ is a bounded and right continuous stationary $\phi$-mixing process with zero mean and mixing rate $\phi(\cdot)$, such that

$$
\int_{0}^{\infty} \phi^{1 / 2}(s) d s<\infty .
$$

(A2) $F(\cdot, \cdot)$ and $F_{x}(\cdot, \cdot)$ are both continuous in $(x, \xi), E F(x, \xi(t))=0$, where $F_{x}(\cdot, \cdot)$ denotes the partial derivative of $F(\cdot, \cdot)$ with respect to $x$. Recall that $x=(y, \dot{y})^{\prime}$ with $z^{\prime}$ denotes the transpose of $z$.

(A3) There are continuous functions $a(\cdot)$ and $b(\cdot)$ such that $a(\cdot)$ is non-negative, and

$$
\begin{gathered}
\int_{s}^{T} E F(x, \xi(u)) F(x, \xi(s)) d u \rightarrow a(x) \\
\int_{s}^{T} E F_{x_{2}}(x, \xi(u)) F(x, \xi(s)) d u \rightarrow b(x)
\end{gathered}
$$

as $T-s \rightarrow \infty$, and $s \rightarrow \infty$.

(A4) There exist random variables $\beta_{i}$ for $i=1,2$, and $\gamma$ such that $\beta_{i}^{\varepsilon} \Rightarrow \beta_{i}$ and $\gamma^{\varepsilon} \Rightarrow \gamma$ as $\varepsilon \rightarrow 0$

These conditions are not restrictive. The $\phi$-mixing processes constitute a large class of processes that have "decreasing dependence" property. In fact, we can work 
with an even larger class of processes that are approximated by functions of $\phi$-mixing processes; see [1]. The weak convergence assumption of $\beta_{i}^{\varepsilon}$ and $\gamma^{\varepsilon}$ are not restrictive either. If $\beta_{i}^{\varepsilon}, \gamma^{\varepsilon}$ are bounded random variables then this condition will be satisfied. If $\beta_{i}^{\varepsilon}, \gamma^{\varepsilon}$ are uniformly integrable, then they are tight, and we can extract convergent subsequences. In fact, our conditions here are more general than the fixed constants auxiliary conditions used in [18]. Before proceeding further, we present a lemma concerning the existence and uniqueness of solution of the boundary value problem (1) and (2).

Lemma 2.1. Assume that conditions of (A1) and (A2) hold, and that for each $\varepsilon>0$, the differential equation (1) has a unique solution for each initial condition. Then the boundary value problem given by the differential equation (1) with boundary condition (2) has a unique solution in the almost sure sense.

Proof. We note that by $(\mathrm{A} 1),\{\xi(\cdot)\}$ is a bounded and right continuous process. By $(\mathrm{A} 2), F(\cdot, \xi)$ is continuous and smooth for each $\xi$ and each fixed $\varepsilon$. Thus for almost all $\omega$, it can be checked that all the conditions in the paper of Lasota and Opia [13, Theorem 1, p.2] are satisfied. As a result, the boundary value problem (1) and (2) has a unique solution almost surely. The lemma is thus proved.

The existence and uniqueness of the solution of the boundary value problem is established for each fixed $\varepsilon>0$. Our task next is to try to approximate the solution of the boundary value problem as $\varepsilon \rightarrow 0$. We proceed to state the main theorem.

Theorem 2.2. Suppose that the conditions of Lemma 2.1 are satisfied, that (A3)(A4) hold, and that (13) has a unique solution (unique in the sense of distribution) for each initial condition. Then the process $\left\{x^{\varepsilon}(\cdot)\right\}$ given by (10) and (11) is tight in $D^{2}[0, \infty)$, and $x^{\varepsilon}(\cdot) \Rightarrow x(\cdot)$ such that $x(\cdot)$ satisfies

$$
\begin{gathered}
\left(\begin{array}{l}
d x_{1}(t) \\
d x_{2}(t)
\end{array}\right)=\left(\begin{array}{l}
x_{2}(t) \\
b(x(t))
\end{array}\right) d t+\left(\begin{array}{ll}
0 & 0 \\
0 & a^{\frac{1}{2}}(x(t))
\end{array}\right)\left(\begin{array}{l}
0 \\
1
\end{array}\right) d w(t), \\
\left(\begin{array}{l}
x_{1}(0) \\
x_{2}(0)
\end{array}\right)=\left(\begin{array}{l}
\beta_{1} \\
\gamma
\end{array}\right),
\end{gathered}
$$

where $w(\cdot)$ is a real-valued standard Brownian motion. Moreover, there exists an $x_{1}(1)=x_{1}(1 ; \gamma)$ satisfying

$$
x_{1}(1)=\beta_{2} \text {. }
$$

Remark 2.3. The theorem above indicates that the solution of problem (10) with initial condition (11) and condition $x_{1}^{\varepsilon}(1)=x_{1}^{\varepsilon}\left(1, \gamma^{\varepsilon}\right)=\beta_{2}^{\varepsilon}$ can be approximated by (13)-(14) together with condition (15). The computation complexity is thus reduced. Similar to the approach of [18], using asymptotic expansion, it is possible to show 
that there exists some $\Delta$ such that a unique solution of the original problem (1)-(2), with shooting parameters within a ball of radius $\Delta$ about those of the limit problem, will exist with probability approaching 1 as $\varepsilon \rightarrow 0$. In what follows, our main effort however is devoted to obtaining limit (13)-(14) using weak convergence methods.

\section{PROOF OF RESULT}

The idea is to show that the limit of $x^{\varepsilon}(\cdot)$ solves a martingale problem with operator

$$
\mathcal{A} f(x)=f_{x}^{\prime}(x)\left(\begin{array}{l}
x_{2} \\
b(x)
\end{array}\right)+\frac{1}{2} \sum_{i j} G_{i j}(x) f_{x_{i} x_{j}}(x)
$$

for any $f(\cdot) \in C_{0}^{2}$, where $f_{x}^{\prime}$ denotes the transpose of $f_{x}$, and $G(x)=\left(\begin{array}{cc}0 & 0 \\ 0 & a(x)\end{array}\right)$. Following [15] and [10], we first define the notion $p$-lim and an operator $\hat{\mathcal{A}}^{\varepsilon}$ as follows.

Let $M$ be the set of real-valued, measurable functions of $(\omega, t)$ that are none zero only on a bounded $t$-interval. Let

$$
\bar{M}^{\varepsilon}=\left\{f \in M ; \sup _{t} E|f(t)|<\infty \text { and } f(t) \text { is } \mathcal{F}_{t}^{\varepsilon} \text { measurable }\right\}
$$

Let $f(\cdot), f^{\delta}(\cdot) \in \bar{M}^{\varepsilon}$, for each $\delta>0, f=p-\lim _{\delta} f^{\delta}$ if and only if

$$
\begin{gathered}
\sup _{t, \delta} E\left|f^{\delta}(t)\right|<\infty \\
\lim _{\delta \rightarrow 0} E\left|f^{\delta}(t)-f(t)\right|=0 \text { for each t. }
\end{gathered}
$$

We say that $f(\cdot) \in \mathcal{D}\left(\hat{\mathcal{A}}^{\varepsilon}\right)$ the domain of $\hat{\mathcal{A}}^{\varepsilon}$, and $\hat{\mathcal{A}}^{\varepsilon} f=g$, if $f, g \in \bar{M}^{\varepsilon}$ and

$$
p \text { - } \lim _{\delta \rightarrow 0}\left(\frac{E_{t}^{\varepsilon} f(t+\delta)-f(t)}{\delta}-g(t)\right)=0 .
$$

To proceed, we shall use the technique developed in [11] and [2]. In particular, we use the perturbed test function methods in what follows. Fix $f(\cdot) \in C_{0}^{2}$ and apply $\hat{\mathcal{A}}^{\varepsilon}$ to $f\left(x^{\varepsilon}(\cdot)\right)$. We are seeking a "small" perturbation $f_{1}^{\varepsilon}(t)$ of $f(\cdot)$, such that $\hat{\mathcal{A}}^{\varepsilon}\left(f\left(x^{\varepsilon}(t)\right)+f_{1}^{\varepsilon}(t)\right)$ is approximated by an appropriate infinitesimal generator of the diffusion.

We also need the notion of $N$-truncation. For each $N>0$, let

$$
S_{N}=\{x ;|x| \leq N\}
$$

be the $N$-ball, and let $x^{\varepsilon, N}(0)=x^{\varepsilon}(0), x^{\varepsilon, N}(t)=x^{\varepsilon}(t)$ up until the first exit from $S_{N}$, and

$$
\lim _{m \rightarrow \infty} \limsup _{\varepsilon} P\left\{\sup _{t \leq T}\left|x^{\varepsilon, N}(t)\right| \geq m\right\}=0 \text { for each } T<\infty
$$


$x^{\varepsilon, N}(\cdot)$ is said to be the $N$-truncation of $x^{\varepsilon}(\cdot)$.

In lieu of (10), we shall consider the following truncated version of the differential equation

$$
\left(\begin{array}{l}
\dot{x}_{1}^{\varepsilon, N}(t) \\
\dot{x}_{2}^{\varepsilon, N}(t)
\end{array}\right)=\left(\begin{array}{l}
x_{2}^{\varepsilon, N}(t) \\
\frac{1}{\varepsilon} F^{N}\left(x^{\varepsilon, N}(t), \xi^{\varepsilon}(t)\right)
\end{array}\right)
$$

where

$$
F^{N}\left(x^{\varepsilon, N}(t), \xi^{\varepsilon}(t)\right)=F\left(x^{\varepsilon, N}(t), \xi^{\varepsilon}(t)\right) q^{N}\left(x^{\varepsilon, N}(t)\right)
$$

such that

$$
q^{N}(z)=\left\{\begin{array}{l}
1, \text { when }|z| \leq N \\
0, \text { when }|z| \geq N+1 .
\end{array}\right.
$$

For ease of notations, we shall write $x$ for $x^{\varepsilon, N}(t)$ whenever is convenient.

It follows that for any $f \in C_{0}^{2}$,

$$
\hat{\mathcal{A}}^{\varepsilon} f(x)=f_{x}^{\prime}(x)\left(\begin{array}{l}
x_{2} \\
\frac{1}{\varepsilon} F\left(x, \xi^{\varepsilon}(t)\right)
\end{array}\right) .
$$

In other word, for our case, $\hat{\mathcal{A}}^{\varepsilon}$ is just a differential operator. Note that $f_{x}(x)$ stands for the gradient of $f(\cdot)$, and $f_{x}^{\prime}(x)=\left(f_{x_{1}}(x), f_{x_{2}}(x)\right)$.

Now, define

$$
f_{1}^{\varepsilon}(t)=\frac{1}{\varepsilon} \int_{t}^{T} E_{t}^{\varepsilon} f_{x}^{\prime}(x)\left(\begin{array}{l}
0 \\
F^{N}\left(x, \xi^{\varepsilon}(u)\right)
\end{array}\right) d u .
$$

$f_{1}^{\varepsilon}(t)$ is introduced to effect the desired cancellation, and to guarantee that the perturbation is small in an appropriate sense. Making change of variable $\frac{u}{\varepsilon^{2}} \rightarrow u$ in (18) yields

$$
f_{1}^{\varepsilon}(t)=\varepsilon \int_{\frac{t}{\varepsilon^{2}}}^{\frac{T}{\varepsilon^{2}}} E_{t}^{\varepsilon} f_{x_{2}}(x) F^{N}\left(x, \xi^{\varepsilon}(u)\right) d u .
$$

By virtue of the strong mixing conditions, the following equations hold for $t<v<u$, (see [11], and references therein).

$$
\begin{gathered}
\left|E_{t} \xi(u) \xi(v)-E \xi(u) \xi(v)\right| \leq 4 \phi^{\frac{1}{2}}(u-v) \phi^{\frac{1}{2}}(v-t) \\
\left|E_{t} \xi(t+s)-E \xi(t+s)\right| \leq 2 \phi(s)
\end{gathered}
$$

where $E_{t}$ denotes the conditioning on $\mathcal{F}_{0}^{t}=\sigma\{\xi(s) ; 0 \leq s \leq t\}$.

In view of (21) and (A1),

$$
\begin{aligned}
\sup _{t \leq T}\left|f_{1}^{\varepsilon}(t)\right| & =\sup _{t \leq T}\left|\varepsilon \int_{\frac{t}{\varepsilon^{2}}}^{\frac{T}{\varepsilon^{2}}} f_{x_{2}}(x) E_{t}^{\varepsilon} F^{N}\left(x, \xi^{\varepsilon}(u)\right) d u\right| \\
& \leq K \varepsilon \sup _{t \leq T}\left(\int_{\frac{t}{\varepsilon^{2}}}^{\frac{T}{\varepsilon^{2}}} \phi\left(u-\frac{t}{\varepsilon^{2}}\right) d u\right) \\
& \rightarrow 0 \text { as } \varepsilon \rightarrow 0 .
\end{aligned}
$$




\section{Define}

$$
f^{\varepsilon}(t)=f(x)+f_{1}^{\varepsilon}(t)
$$

Since

$$
\begin{aligned}
\hat{\mathcal{A}}^{\varepsilon} f_{1}^{\varepsilon}(t)= & -\frac{1}{\varepsilon} f_{x_{2}}(x) F^{N}\left(x, \xi^{\varepsilon}(t)\right) \\
& +\varepsilon \int_{\frac{t}{\varepsilon^{2}}}^{\frac{T}{\varepsilon^{2}}} E_{t}^{\varepsilon}\left(f_{x_{2}}(x) F^{N}(x, \xi(u))\right)_{x}^{\prime} x^{\varepsilon, N}(t) d u, \\
\hat{\mathcal{A}}^{\varepsilon} f^{\varepsilon}(t)= & f_{x_{1}}(x) x_{2}+g^{\varepsilon}(t) \\
& +\int_{\frac{t}{\varepsilon^{2}}}^{\frac{T}{\varepsilon^{2}}} E\left(f_{x_{2}}(x) F^{N}(x, \xi(u))\right)_{x}^{\prime}\left(\begin{array}{l}
\varepsilon x_{2} \\
F^{N}\left(x, \xi^{\varepsilon}(t)\right)
\end{array}\right),
\end{aligned}
$$

with

$$
\begin{aligned}
g^{\varepsilon}(t)= & \int_{\frac{t}{\varepsilon^{2}}}^{\frac{T}{\varepsilon^{2}}}\left\{E_{t}^{\varepsilon}\left(f_{x_{2}}(x) F^{N}(x, \xi(u))\right)_{x}^{\prime}\left(\begin{array}{l}
\varepsilon x_{2} \\
F^{N}\left(x, \xi^{\varepsilon}(t)\right)
\end{array}\right)\right. \\
& \left.-E\left(f_{x_{2}}(x) F^{N}(x, \xi(u))\right)_{x}^{\prime}\left(\begin{array}{l}
\varepsilon x_{2} \\
F^{N}\left(x, \xi^{\varepsilon}(t)\right)
\end{array}\right)\right\} d u .
\end{aligned}
$$

By virtue of $(20), p-\lim _{\varepsilon} g^{\varepsilon}(t)=0$.

As for the third term on the right-hand side of (25),

$$
\begin{aligned}
& \int_{\frac{t}{\varepsilon^{2}}}^{\frac{T}{\varepsilon^{2}}} E\left(f_{x_{2}}(x) F^{N}(x, \xi(u))\right)_{x}\left(\begin{array}{l}
\varepsilon x_{2} \\
F^{N}\left(x, \xi^{\varepsilon}(t)\right)
\end{array}\right) d u \\
& =\int_{\frac{t}{\varepsilon^{2}}}^{\frac{T}{\varepsilon^{2}}} E f_{x_{2} x_{2}}(x) F^{N}(x, \xi(u)) F^{N}\left(x, \xi^{\varepsilon}(t)\right) d u \\
& \quad+\int_{\frac{t}{\varepsilon^{2}}}^{\frac{T}{\varepsilon^{2}}} E f_{x_{2}}(x) F_{x_{2}}^{N}(x, \xi(u)) F^{N}\left(x, \xi^{\varepsilon}(t)\right) d u \\
& \quad+\varepsilon \int_{\frac{t}{\varepsilon^{2}}}^{\frac{T}{\varepsilon^{2}}} E f_{x_{1} x_{2}}(x) x_{2} F^{N}(x, \xi(u)) d u \\
& \quad+\varepsilon \int_{\frac{t}{\varepsilon^{2}}}^{\frac{T}{\varepsilon^{2}}} E f_{x_{2}}(x) x_{2} F_{x_{1}}^{N}(x, \xi(u)) d u .
\end{aligned}
$$

In view of (A3), the first term on the right-hand side of $(27)$ tends to $f_{x_{2} x_{2}}(x) a(x)$, and the second term tends to $f_{x_{2}}(x) b(x)$. For the third term, by the strong mixing,

$$
\begin{aligned}
\varepsilon \int_{\frac{t}{\varepsilon^{2}}}^{\frac{T}{\varepsilon^{2}}} E f_{x_{1} x_{2}}(x) x_{2} F^{N}(x, \xi(u)) d u & =\varepsilon \int_{\frac{t}{\varepsilon^{2}}}^{\frac{T}{\varepsilon^{2}}} E f_{x_{1} x_{2}}(x) x_{2} E_{t}^{\varepsilon} F^{N}(x, \xi(u)) d u \\
& \rightarrow 0 \text { as } \varepsilon \rightarrow 0 .
\end{aligned}
$$

Similarly, the last term tends to 0 as $\varepsilon \rightarrow 0$.

Consequently, $\left\{\hat{\mathcal{A}}^{\varepsilon} f^{\varepsilon}(\cdot) ; t>0, t \leq T\right\}$ is uniformly integrable for each $f \in C_{0}^{2}$, and

$$
\lim _{\varepsilon} P\left\{\sup _{t \leq T}\left|f^{\varepsilon}(t)-f\left(x^{\varepsilon, N}(t)\right)\right| \geq m\right\}=0 \text { for each } m>0
$$


By Theorem 3.3.4 in [11], we conclude that $\left\{x^{\varepsilon, N}(\cdot)\right\}$ is tight. By virtue of the above result and (A1)

$$
p-\lim _{\varepsilon}\left(\hat{\mathcal{A}}^{\varepsilon} f^{\varepsilon}(t)-\hat{\mathcal{A}}^{\varepsilon} f\left(x^{\varepsilon, N}(t)\right)\right)=0 .
$$

It now follows from Theorem 3.3 .2 in [11], $x^{\varepsilon, N}(\cdot) \Rightarrow x^{N}(\cdot)$.

Let $P(\cdot)$ and $P^{N}(\cdot)$ be the measures induced by $x(\cdot)$ and $x^{N}(\cdot)$ respectively, on the Borel subsets of $D^{r}[0, \infty)$. The measure $P(\cdot)$ is unique, since the corresponding martingale problem or the SDE has a unique solution. Now, for any $T<\infty$, let $H$ be any Borel subset of the set of paths in $D^{r}[0, \infty)$ with values in $S_{N}, P(H)=P^{N}(H)$, But $P\left\{\sup _{t \leq T}|x(t)| \leq N\right\} \rightarrow 1$ as $N \rightarrow \infty$. This together with $x^{\varepsilon, N}(\cdot) \Rightarrow x^{N}(\cdot)$ imply that $x^{\varepsilon}(\cdot) \Rightarrow x(\cdot)$. Finally, looking at equation (12), the left-hand side converges weakly to $x_{1}(1)$, and the right-hand side converges to $\beta_{2}$. The proof of the theorem is thus complete.

\section{EXAMPLES}

Some examples are given in this section. The verification of the statements is a straight forward application of our theorem. In what follows, $w$ is a standard Brownian motion. The $F(\cdot), F_{0}(\cdot)$, and $F_{1}(\cdot)$ are all continuous functions, and

$$
\left(\begin{array}{l}
x_{1} \\
x_{2}
\end{array}\right)=\left(\begin{array}{c}
y \\
\dot{y}
\end{array}\right) \text {. }
$$

Example 4.1. Consider

$$
\begin{gathered}
\ddot{y}^{\varepsilon}(t)=F\left(y^{\varepsilon}(t)\right)+\frac{1}{\varepsilon} \xi^{\varepsilon}(t) \\
y^{\varepsilon}(0)=0, y^{\varepsilon}(1)=1 .
\end{gathered}
$$

Suppose $\xi^{\varepsilon}(t)=\xi\left(\frac{t}{\varepsilon^{2}}\right)$, and $\xi(\cdot)$ is a right continuous stationary random process, satisfying (A1). Suppose that $E F(x, \xi(t))=0$, and $E(\xi(t))^{2}=R^{2}$ for some $R>0$. then as $\varepsilon \rightarrow 0,\left(\begin{array}{c}x_{1}^{\varepsilon}(\cdot) \\ x_{2}^{\varepsilon}(\cdot)\end{array}\right) \Rightarrow\left(\begin{array}{c}x_{1}(\cdot) \\ x_{2}(\cdot)\end{array}\right)$, and

$$
\left(\begin{array}{l}
d x_{1}(t) \\
d x_{2}(t)
\end{array}\right)=\left(\begin{array}{l}
x_{2}(t) \\
F\left(x_{1}(t)\right)
\end{array}\right) d t+\left(\begin{array}{ll}
0 & 0 \\
0 & R
\end{array}\right)\left(\begin{array}{l}
0 \\
1
\end{array}\right) d w(t),
$$

where $w(\cdot)$ is a standard real-valued Brownian motion,

$$
x_{1}(0)=0, x_{2}(0)=\gamma
$$

and there is an $x_{1}(1 ; \gamma)$ satisfying $x_{1}(1 ; \gamma)=1$. 
Example 4.2. Consider

$$
\begin{gathered}
\ddot{y}^{\varepsilon}(t)=F_{0}\left(y^{\varepsilon}(t)\right)+\frac{1}{\varepsilon} F_{1}\left(y^{\varepsilon}(t)\right) \xi^{\varepsilon}(t) \\
y^{\varepsilon}(0)=0, y^{\varepsilon}(1)=1 .
\end{gathered}
$$

Let $\xi(\cdot)$ satisfy the same conditions as in Example 4.1. Then $\left(\begin{array}{c}x_{1}^{\varepsilon}(\cdot) \\ x_{2}^{\varepsilon}(\cdot)\end{array}\right) \Rightarrow\left(\begin{array}{c}x_{1}(\cdot) \\ x_{2}(\cdot)\end{array}\right)$, with

$$
\left(\begin{array}{l}
d x_{1}(t) \\
d x_{2}(t)
\end{array}\right)=\left(\begin{array}{l}
x_{2} \\
F_{0}\left(x_{1}(t)\right)
\end{array}\right) d t+\left(\begin{array}{ll}
0 & 0 \\
0 & R F_{1}\left(x_{1}(t)\right)
\end{array}\right)\left(\begin{array}{l}
0 \\
1
\end{array}\right) d w(t)
$$

where $w(\cdot)$ is a standard real-valued Brownian motion,

$$
x_{1}(0)=0, x_{2}(0)=\gamma
$$

and there is an $x_{1}(1 ; \gamma)$ satisfying $x_{1}(1 ; \gamma)=1$.

Example 4.3. In particular, if in Example 4.2, $F_{0}(y)=-K_{1}^{2} y$, for some $K_{1}>0$, and $F_{1}(y)=y$, then (34) becomes the well-known Helmholtz equation

$$
\ddot{y}^{\varepsilon}(t)=-K_{1}^{2} y^{\varepsilon}(t)+\frac{1}{\varepsilon} y^{\varepsilon}(t) \xi^{\varepsilon}(t) .
$$

The limit is then given as the solution of

$$
\left(\begin{array}{l}
d x_{1}(t) \\
d x_{2}(t)
\end{array}\right)=\left(\begin{array}{l}
x_{2}(t) \\
-K_{1}^{2} x_{1}(t)
\end{array}\right) d t+\left(\begin{array}{ll}
0 & 0 \\
0 & R x_{1}(t)
\end{array}\right)\left(\begin{array}{l}
0 \\
1
\end{array}\right) d w(t)
$$

where $w(\cdot)$ is a real-valued standard Brownian motion.

\section{CONCLUDING REMARKS}

We conclude this paper by noting the following points.

(a) A limit result associated with a nonlinear differential equation with wide-band noise perturbations and random boundary conditions is obtained in this paper. A diffusion limit is derived by using weak convergence methods.

(b) This result leads to an approximation to the two-point boundary value problem (1) and (2). If $\varepsilon$ is sufficiently small, in lieu of solving the original problem, we can find solutions for the limit problem and use the limit as an approximation. 
(c) Equation (10) is generally nonlinear. Since random variables are involved, typical numerical schemes for searching roots of nonlinear equations will not work. However, the methods of stochastic approximation can be applied. Stochastic approximation is a well studied subject with a huge literature. For most recent development, see [12] and references therein. We shall not dwell on it here.

(d) We proved the limit theorem under the assumption that the noise satisfies mixing property. Other noise models can be considered too. for example, if $\xi(t)=\int_{-\infty}^{t} h(t-s) d w(s)$ and $\xi^{\varepsilon}(t)=\xi\left(\frac{t}{\varepsilon^{2}}\right)$, with $h(t) \rightarrow 0$ exponentially as $t \rightarrow \infty$; and $F(x, \xi)=F_{1}(x) \xi$ with $F_{1}(\cdot) \in C^{2}$, then the limit theorem still holds with minor modifications.

(e) For recent results on two-time-scale diffusions and Markovian systems, we refer the reader to $[7,8,9,19]$, and the references therein. It is possible to extend the results of this paper to the case that there is an additional continuous-time Markov chain. The resulting systems will be switching diffusions. We refer the reader to [20] but omit the details.

\section{ACKNOWLEDGEMENT}

Research of G. Yin was supported in part by the Army Research Office under Grant W911NF-15-1-0218.

\section{REFERENCES}

[1] P. Billingsley, Convergence of Probability Measures, J. Wiley \& Sons, 1968.

[2] G. Blankenship and G. Papanicolaou, Stability and control of stochastic systems with wide band noise, SIAM, J. Appl. Math. 34 (1978), 437-476.

[3] S. Ethier and T.G. Kurtz, Markov Processes, J. Wiley \& Sons, 1986.

[4] L. Jackson, Existence and uniqueness of solutions of boundary value problems for third order differential equations, 13 (1973), 432-437.

[5] H.B. Keller, Numerical Methods for Two-Point Boundary-Value Problems, Blaisdell Pub. Co., 1968.

[6] R.Z. Khasminskii, Stochastic process defined by differential equations, Theory Probab. Appl., 11 (1966), 211-228.

[7] R.Z. Khasminskii and G. Yin, On transition densities of singularly perturbed diffusions with fast and slow components, SIAM J. Appl. Math., 56 (1996), 1794-1819. 
[8] R.Z. Khasminskii and G. Yin, On averaging principles: An asymptotic expansion approach, SIAM J. Math. Anal., 35 (2004), 1534-1560.

[9] R.Z. Khasminskii and G. Yin, Limit behavior of two-time-scale diffusions revisited, J. Differential Eqs., 212 (2005) 85-113.

[10] T. Kurtz, Semigroups of conditional shifts and approximation of Markov processes, Ann. Probab., 4 (1975), 618-642.

[11] H.J. Kushner, (1984) Approximation and Weak Convergence Methods for Random Processes with Applications to Stochastic Systems Theory, MIT Press, Cambridge, MA, 1984.

[12] H.J. Kushner and G. Yin, Stochastic Approximation and Recursive Algorithms and Applications, 2nd ed., Springer-Verlag, New York, NY, 2003.

[13] A. Lasota and Z. Opial, On the existence and uniqueness of solutions of a boundary value problem for an ordinary second order differential equation, Colloq. Math. 18 (1967), 1-5.

[14] D. Nualart and E. Pardoux, Boundary value problems for stochastic differential equations, Ann. Probab., 19 (1991), 1118-1144

[15] R. Rishel, Necessary and sufficient conditions for continuous stochastic optimal control, SIAM J. Control Optim., 8 (1970), 559-571.

[16] N.M. Xia, The density function of the solution of a two-point boundary value problem containing small stochastic processes, Quar. Appl. Math., XLVI (1988), 29-47.

[17] N.M. Xia, W.E. Boyce, and M.R. Barry, Two point boundary value problems containing a finite number of random variables, Stoch. Anal. Appl., 1 (1983), $117-137$.

[18] B.S. White and J.N. Franklin, A limit theorem for stochastic two point boundary value problems of ordinary differential equations, Comm. Pure Appl. Math., 32 (1979), 253-275.

[19] G. Yin and Q. Zhang, Continuous-Time Markov Chains and Applications: A Two-Time-Scale Approach, 2nd Ed., Springer, New York, 2013.

[20] G. Yin and C. Zhu, Hybrid Switching Diffusions: Properties and Applications, Springer, New York, 2010. 
